cases of histamine release induced bronchospasm and hypotension have been reported to the Committee on Safety of Medicines.

Although in the past the combination of fentanyl, halothane, and curare has been noted for producing episodes of bradycardia, they do not seem to have been as profound or to have caused such concomitant changes in the QRS complexes as those observed with atracurium. There are two possibilities for this phenomenon: firstly, atracurium's lack of cardiovascular effects may mean that the cholinergic actions of other anaesthetic agents are unattenuated, and, secondly, perhaps a metabolite is having an effect. Laudanosine, the main metabolite, ${ }^{2}$ has a structure similar to apomorphine and may be contributory to these electrocardiographic changes.

Whatever the cause, I consider that it would be advisable in all operations in which vagal stimulation is expected that the patients undergo full electrocardiographic monitoring and that atropine should be given as part of the premedication or induction sequence.

\section{References}

${ }^{1}$ Payne JP, Hughes R. Evaluation of atracurium in anaesthetised man. $\mathrm{Br} \mathcal{F}$ Anaesth $1981 ; 53: 45-54$.

2 Stenlake JB. Ions-cyclic nucleotides-cholinergy. In: Stoclet JC, ed. Advances in pharmacology and therapeutics. Oxford and New York: Pergamon Press, 1979:303.

${ }^{3}$ Hughes R, and Chapple DJ. Pharmacology of atracurium. Br $\mathcal{J}$ Anaesth $1981 ; 53: 31-44$.

(Accepted 11 May 1983)

\title{
Recovery of renal function after prolonged dialysis and transplantation
}

\author{
T O NUNAN, E A STEVENS, D N CROFT, P J HILTON, N F JONES, A J WING
}

\begin{abstract}
Out of 250 patients with renal failure, seven $(2 \cdot 8 \%)$ treated by regular haemodialysis alone (four) or given cadaveric allografts (three) later showed recovery of function of their own kidneys lasting from one to four years. In the patients receiving haemodialysis alone recovery was easily recognised from their serum creatinine concentrations, but in those with transplants recovery was discovered unexpectedly during radionuclide scanning.

These findings suggest that recovery of renal function may be more common than generally recognised, which should be borne in mind when beginning renal replacement treatment and particularly when contemplating bilateral nephrectomy.
\end{abstract}

\section{Introduction}

Recovery of renal function in patients receiving long term dialysis or after renal transplantation has been reported. ${ }^{12}$ The registry of the European Dialysis and Transplant Association has collected 573 patients from a total of 91866 who had been treated by dialysis for more than six weeks in whom recovery lasted more than three months-an incidence of $0.6 \% .^{2}$ In our renal unit recovery occurred in seven of the first 250 patients $(2 \cdot 8 \%)$ accepted for renal replacement therapy. Recovery of renal function was proved by radionuclide imaging in three patients who had received kidney transplants. Without isotope scanning recovery of function by the patients' own kidneys would not have been detected.

\footnotetext{
Departments of Renal and Nuclear Medicine, St Thomas's Hospital, London SE1 7EH

T O NUNAN, MSC, MRCP, senior medical registrar

E A STEVENS, SRN, HV, nursing officer

D N CROFT, DM, FRCP, consultant physician

P J HILTON, MD, FRCP, consultant physician

N F JONES, MD, FRCP, consultant physician

A J WING, DM, FRCP, consultant physician

Correspondence to: Dr T O Nunan.
}

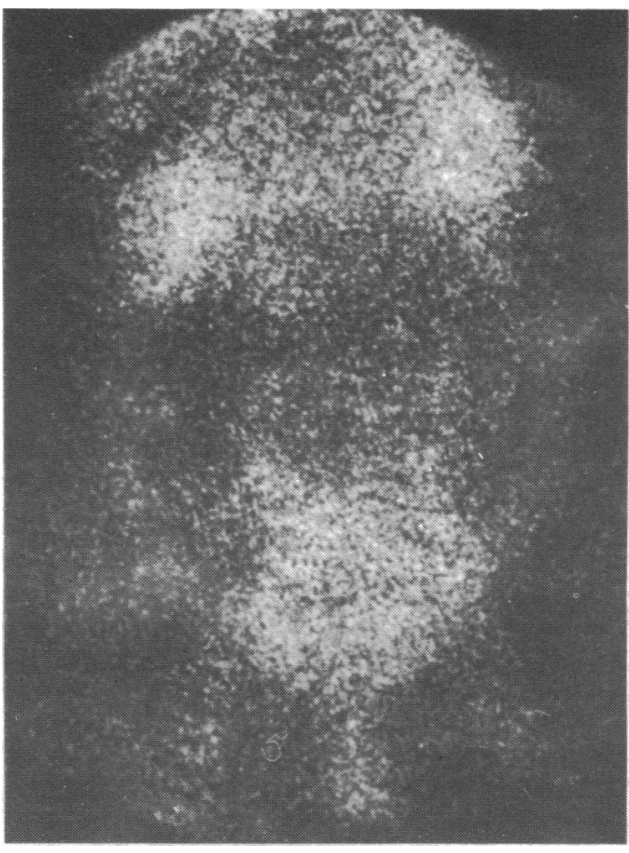

${ }^{99 m}$ Tc-DTPA gammacamera scan of transplant recipient with crescentic glomerulonephritis showing uptake by her own two kidneys.

\section{Present series}

Four of the seven patients we describe had received regular haemodialysis as the only form of renal replacement therapy. Their primary renal diseases were mesangiocapillary glomerulonephritis in two, crescentic glomerulonephritis in one, and Goodpasture's syndrome in one. The periods of dialysis before detection of recovery of renal function were $13,41,13$, and 12 weeks respectively. In the two patients with mesangiocapillary glomerulonephritis recovery of renal function was sufficient to remove the need for dialysis for 50 and 41 weeks respectively. The patient with crescentic glomerulonephritis remained well for four years with a serum creatinine concentration of $380 \mu \mathrm{mol} / 1(4.3 \mathrm{mg} / 100 \mathrm{ml})$ or less before her renal disease recurred and dialysis again became necessary. The patient with Goodpasture's 
syndrome was well three and a half years after termination of dialysis with a serum creatinine concentration of $156 \mu \mathrm{mol} / 1(1.8 \mathrm{mg} / 100 \mathrm{ml})$.

Three patients had received cadaveric renal transplants and conventional immunosuppressive treatment. Two had accelerated hypertension and one crescentic glomerulonephritis. Radionuclide studies in the immediate postoperative period confirmed that the transplants were functioning in all cases. Further studies using ${ }^{99 \mathrm{~m}} \mathrm{Tc}$ labelled diethylenetriamine penta-acetic acid (DTPA) 22, 72, and 278 weeks later respectively proved that each of the three grafts had stopped functioning and that the patients' own kidneys were functioning (figure). This finding was not anticipated in any of these patients when the scan was arranged. The two patients with accelerated hypertension had remissions lasting 64 and 74 weeks before they again became uraemic. The third patient remained well with a plasma creatinine concentration of $77 \mu \mathrm{mol} / 1(0.9 \mathrm{mg} / 100 \mathrm{ml})$ two and a half years after the discovery that her transplant was not functioning.

Immunosuppressive treatment was withdrawn in the two patients given transplants for accelerated hypertension when recovery of their own renal function was detected. The two patients with mesangiocapillary glomerulonephritis were given immunosuppressive treatment when renal function fell again after the original recovery, but no benefit resulted. The patient with Goodpasture's syndrome had been treated by plasmapheresis in the months before dialysis, and immunosuppressive treatment was restarted when recovery occurred. Of the two patients with crescentic glomerulonephritis, one given a renal transplant continued immunosuppressive treatment, and one treated by regular haemodialysis received immunosuppressive treatment again when recovery occurred.

\section{Discussion}

The high incidence of recovery of renal function in our series $(2.8 \%)$ suggests that recovery may occur more frequently than is recognised. Unnecessary dialysis is clearly undesirable. Recovery of renal function is relatively easy to detect in patients treated by dialysis. In the presence of a renal transplant evidence of recovery of function of the patient's own kidneys by radionuclide imaging has not previously been described. We emphasise that neither recovery of host renal function nor loss of graft function had been detected before the scans were performed.

Our three patients with transplants whose own kidneys recovered function amounted to about $5 \%$ of our patients alive with functioning grafts more than one year after operation. Similar experience in other centres would make it necessary to question the validity of long term transplant survival statistics. There may be a place for routine renal scanning of all patients with kidney grafts after perhaps one and two years, even when overall renal function is satisfactory. The possibility of recovery of function, albeit remote, must also be considered whenever a patient begins renal replacement treatment and particularly if bilateral nephrectomy is contemplated.

Whether immunosuppressive treatment should be continued or reinstituted after recovery of host kidney function depends on the primary renal disease. We stopped immunosuppressive treatment in those patients with accelerated hypertension, but continued it in patients with primary, glomerulonephritis.

\section{References}

${ }^{1}$ Kopsa H, Schmidt P, Zazgornik J, Pils P, Balke P. Recovery of renal function following prolonged acute renal failure. Proc Eur Dial Transplant Assoc 1979;15:495-9.

2 Brynger H, Brunner FP, Chantler C, et al. Combined report on regular dialysis and transplantation in Europe. X, 1979. Proc Eur Dial Transplant Assoc 1981;17:2-86.

\title{
Attempted suicide by insulin injection treated with artificial pancreas
}

\author{
H GIN， B LARNAUDIE, J AUBERTIN
}

\begin{abstract}
An elderly woman with longstanding insulin dependent diabetes tried to commit suicide by injecting 400 units of insulin subcutaneously (usual total daily dose 56 units). She was admitted to hospital within the hour and treated with the aid of an artificial pancreas. This avoided the usual difficulty of the physician having to cope with rapid and substantial fluctuations in blood glucose concentrations and 67 hours after the overdose insulin was reinstituted.

Using an artificial pancreas in insulin overdose is an important advance in management and may avoid the need for surgical intervention such as excising the site of injection.
\end{abstract}

\footnotetext{
Clinique Médicale et des Maladies infectieuses, Hôpital Pellegrin, 33076 Bordeaux Cedex, France

H GIN, MD, lecturer in internal medicine

B LARNAUDIE, MD, lecturer in internal medicine

J AUBERTIN, MD, professor of internal medicine and infectious diseases

Correspondence to: Dr H Gin.
}

\section{Introduction}

Attempted suicide by injection of insulin is rare, even in diabetics. ${ }^{1}$ In all cases, however, hypoglycaemic coma ensues which may be followed by death, hypoglycaemic encephalopathy, or recovery, depending on how soon normoglycaemia is restored. Treatment consists in rousing the patient by means of glucagon injection or glucose injection or both, then keeping him awake and alert by maintaining an adequate intake and avoiding both an overload of glucose and insulin deficiency. Finally, usual insulin treatment is resumed.

Though with close medical supervision such treatment is perfectly practicable, it is often complicated by rapid and substantial changes in the blood glucose concentration. An artificial pancreas, however, provides continuous blood glucose analysis and automatically modulates glucose perfusion. Using this device allows insulin to be resumed as soon as necessary and may avoid the need for such measures as excising the site of injection. ${ }^{2}$

\section{Case history}

A 70 year old woman had a history of insulin dependent diabetes since the age of 18, for which she was receiving a total of 56 units of 